\title{
First-Principle Study for Influence of External Strain on Electronic Structure and Optical Properties of $\delta$-SnSe
}

\author{
M. LUO ${ }^{a}$ AND H.H. YIN ${ }^{b, *}$ \\ ${ }^{a}$ Department of Physics, Shanghai Polytechnic University, Shanghai 201209, China \\ ${ }^{b}$ Department of Electronic Engineering, Shang Hai Jian Qiao University, \\ Shanghai 201306, China
}

Received: 21.03.2020 \& Accepted: 23.06.2020

Doi: 10.12693/APhysPolA.138.504

*e-mail: hhyin81@gmail.com

\begin{abstract}
Electronic and optical properties of $\delta$-SnSe with different strains are investigated through first-principles calculations. It is shown that under a tensile strain, the band gap of $\delta$-SnSe changes from indirect to direct, however its value remains the same. For comparison, when a compressive strain is applied, not only an indirect to direct transformation of the band gap is observed but also gradually reduction of its value to $1.26 \mathrm{eV}$. Further the density of electronic states is studied in these systems. The Sn $p$ and Se $p$ states at the bottom of the conduction band contribute to variations of the band structures. Moreover, the absorption strength in visible light has been enhanced due to the application of an external strain. These results show that the strained $\delta$-SnSe might provide some potential applications in spintronic devices and optical fields.
\end{abstract}

topics: SnSe strain, tunable gap, optical, DFT calculations

\section{Introduction}

As the leading light of two-dimensional (2D) materials, graphene has some remarkable properties, such as ultrathin nature, transparency, flexibility, strong interactions with lights, high carrier mobility, etc. [1-3]. The discovery and the application of graphene has inspired physicists to investigate on other $2 \mathrm{D}$ material. This interest is ongoing due to invaluable opportunities of these materials for innovative applications, such as silicene [4], germanane [5], arsenene [6] and phosphorene [7]. As described in [8-11], the applications of IV, III-V, and IV-VI semiconductors have been extremely expanded. One of an important IV-VI semiconductor compound is SnSe which has an orthorhombic and double-layered structure with weak van der Waals interaction [12-14]. In the past few years, SnSe has been widely used in many areas, such as optoelectronic devices, memory devices, and lithium cells [15-18].

More recently, tunable band-gap materials are shown to significantly widen the light absorption range of solar cell devices, especially the absorption of infrared light [19] by which the efficiency of energy conversion is improved. The successful synthesis of SnSe monolayer or ultrathin film is reported [20] with the band-gap greater than that of the bulk structure [21], and for single layer SnSe, the band gap can be transformed from indirect to direct and vice versa [22]. The tunable band gap of thin film or monolayer SnSe [23] suggests a prospective application in better sun light absorption for solar energy conversion. One of the most popular methods to modify the band gap of a monolayer is by strain engineering [24-26]. To our knowledge, few studies have been reported on the electronic and optical properties of $\delta$-SnSe under strain. Therefore, in this paper, we systematically investigate the electronic and optical properties of $\delta$-SnSe. Firstly, we calculate band structures of $\delta$-SnSe under different strains. The band gap stays stable under a tensile strain, while it becomes tunable when applying a compressive strain. Then, we study the effect of strain on the optical properties of $\delta$-SnSe. Our results can provide a promising platform for exploring the possible applications of $\delta$-SnSe in the spintronic and optical fields.

\section{Method}

All the calculations of material properties are performed with the Vienna ab initio Simulation Package (VASP) [27]. The exchange-correlation potential is treated by using the generalized gradient approximation (GGA) of the Perdew-Burke-Ernzerhof (PBE) functional $[28,29]$. The cutoff energy is set at $450 \mathrm{eV}$. The SnSe monolayer is constructed in the ac-plane, and a vacuum thickness of $20 \AA$ is adopted along the $\mathbf{b}$-direction to avoid interaction between two adjacent periodic images. For the orthorhombic $\delta$-SnSe monolayer, the structural data is listed in Table I. The geometric structure is shown in Fig. 1. The projectoraugmented wave 
(a)

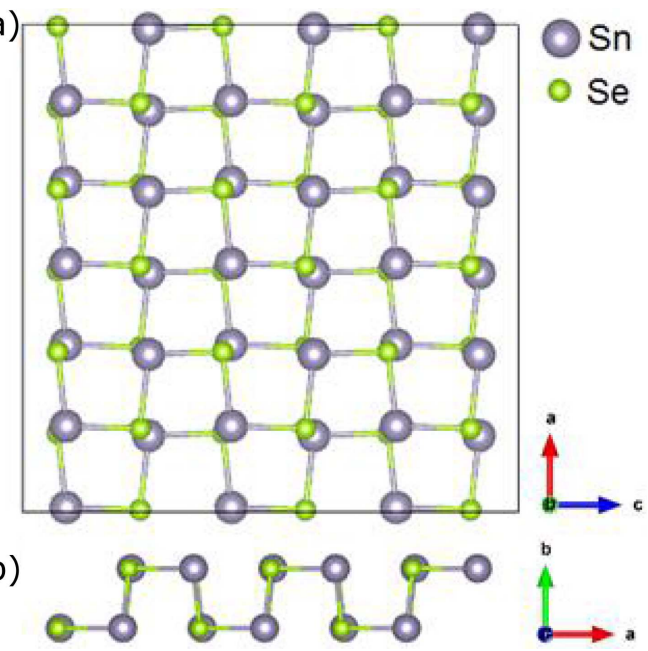

Fig. 1. Schematic structure showing (a) top and (b) side views of pristine $3 \times 1 \times 3 \delta$-SnSe.

TABLE I

Structural data of $\delta$-SnSe belonging to material space group Pca21 (29). Lattice parameters: $a=6.14 \AA$, $c=6.23 \AA, \alpha=\beta=\gamma=90^{\circ}$.

\begin{tabular}{c|c|c|c|c|c}
\hline \hline $\begin{array}{c}\text { Wyckoff } \\
\text { positions }\end{array}$ & Atoms & Site & $x / a$ & $y / b$ & $z / c$ \\
\hline $4 \mathrm{a}$ & Sn1 & 1 & 0.97027 & 0.56204 & 0.76183 \\
$4 \mathrm{a}$ & Se1 & 1 & 0.48587 & 0.43773 & 0.21076
\end{tabular}

potentials including 4 valence electrons for $\operatorname{Sn}\left(s^{2} p^{2}\right)$ and 6 electrons for Se $\left(s^{2} p^{4}\right)$ are considered. The Brillouin zone (BZ) is sampled by a MonkhorstPack $k$-mesh of $5 \times 1 \times 5$. All the structures are optimized until the force and total energy are set at $0.01 \mathrm{eV} \AA$ and $10^{-5} \mathrm{eV}$, respectively.

\section{Results and discussion}

\subsection{Electronic properties of $\delta$-SnSe under strain}

In order to understand how the strain affects the electronic properties of $\delta$-SnSe, we calculate the band structures of $3 \times 1 \times 3 \delta$-SnSe monolayer under various biaxial strains. Here, the value of the strain upon the system is from $-10 \%$ to $10 \%$.

A positive value indicates a tensile strain and a negative value designates a compressive strain. As shown in Fig. 2, the band gap varies very little under the tensile strain, but it is obviously reduced under the compressive strain. In order to provide more details, the corresponding band structures are plotted in Fig. 3. The unstrained $\delta$-SnSe is a semiconductor with an indirect band gap of $2.23 \mathrm{eV}$ (see Fig. 3a), which is consistent with theoretical work [30]. Our calculations showed that the tensile strain, ranging from $0 \%$ to $10 \%$, has little effect on the band structures. As shown in Fig. 3b and 3c, the value of the band gap remains nearly the same,

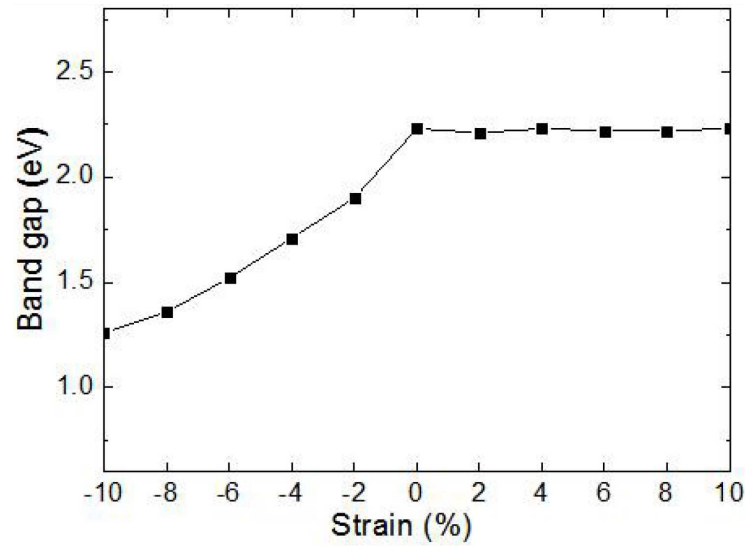

Fig. 2. The band gap of $3 \times 1 \times 3 \delta$-SnSe under different strains. (a) $0 \%$

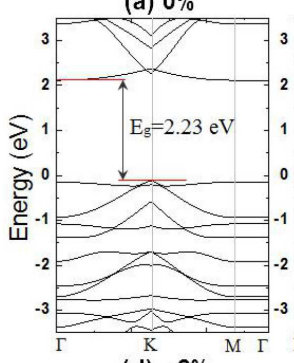

(d) $-2 \%$

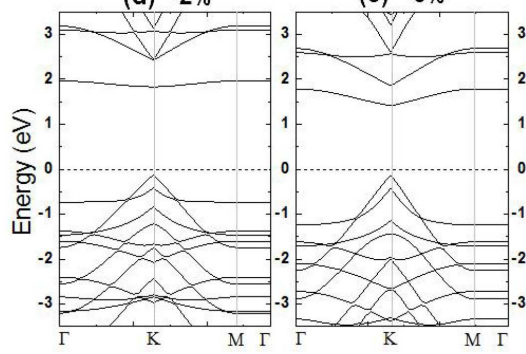

(b) $6 \%$

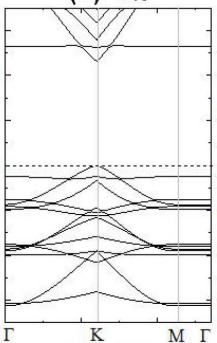

(e) $-6 \%$

(c) $10 \%$
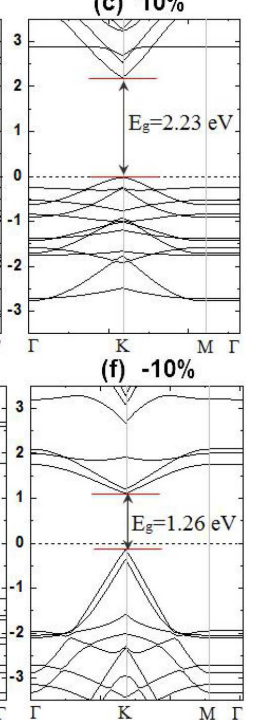

Fig. 3. Band structures of (a) the unstrained $\delta$ SnSe, and of (b)-(f) $\delta$-SnSe under different strains, where the Fermi level is indicated by the dotted line.

however a direct band gap appears at $K$-point. In fact, the compressive strain has much more effect on the band structures (Fig. 3d-f). One can clearly see that the band gap decreases gradually, and a minimum value of $1.26 \mathrm{eV}$ is observed at the $-10 \%$ strain. Some changes take place in both the conduction band $(\mathrm{CB})$ and the valence band (VB). Under the compressive strain, the $\mathrm{CB}$ moves to the Fermi level $\left(E_{\mathrm{F}}\right)$, but the VB stays unchanged. Noteworthy, the gap changes from indirect to direct at $K$-point. By comparison, we can see that the band structure of $\delta$-SnSe is more sensitive to the compressive strain, and thus, an adjustable band gap could be achieved.

The total density of states (TDOS) and the projected density of states (PDOS) of Sn and Se atoms under different strains values, i.e., $10 \%, 6 \%, 0 \%$, $-2 \%,-10 \%$, are presented in Fig. 4 . The purpose is to analyze the effect of strain on the electronic 


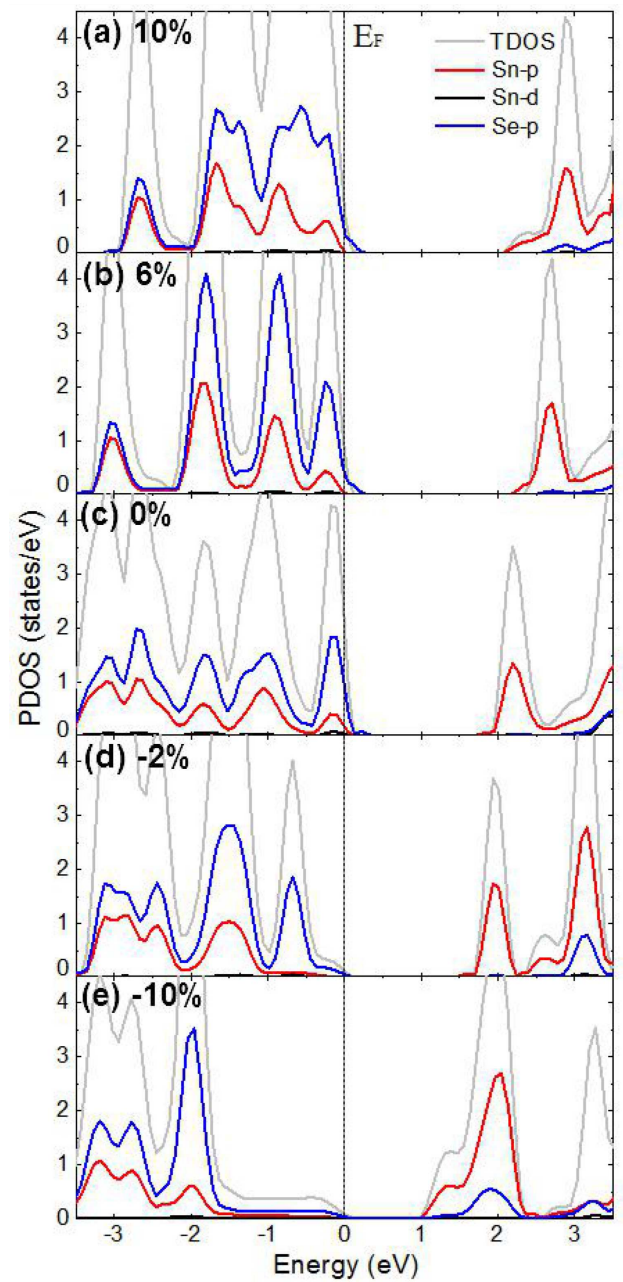

Fig. 4. PDOS of $\delta$ SnSe under (a) $10 \%$, (b) $6 \%$, (c) $0 \%,(\mathrm{~d})-2 \%$, and (e) $-10 \%$ strain. The $0 \%$ strain denotes the unstrained state of $\delta \mathrm{SnSe}$.

structure. Therefore, one can see in Fig. $4 \mathrm{a}$ and $4 \mathrm{~b}$ that under the tensile strain the VB of the system is contributed by both $\mathrm{Sn} p$ and Se $p$ states. In turn, the $\mathrm{CB}$ is only contributed by $\mathrm{Sn} p$, while Sn $d$ state has little contribution. In Fig. 4c one can note that the VB of the unstrained SnSe is contributed by Sn $p$ and Se $p$ states, while the CB is only contributed by $\mathrm{Sn} p$. The composition of CB is the same when a weak compressive strain values of about $-2 \%$ is applied, but in case of $\mathrm{VB}$ composition only Se $p$ state contributes (Fig. 4d). Importantly, when the tensile strain becomes large, namely, equal $-10 \%$, the situation changes again. Then, the composition of $\mathrm{VB}$ is formed by Se $p$ state, and the CB is composed of Sn $p$ and Se $p$ states (see Fig. 4e).

\subsection{Optical properties of $\delta$-SnSe under strain}

Considering that $\delta$-SnSe is a semiconductor with a potential for application in the optical field, we investigate the optical properties of $\delta$-SnSe under different strain, the real part of the dielectric function, and the imaginary part of the dielectric function have been studied systematically. The dielectric function $\varepsilon(\omega)$ establishes a relationship between the electron structure of a solid and the microscopic physical process of electron transition. Moreover, it reflects the band structure and spectral information of the solid. For $\varepsilon(\omega)$ description of one can use the following complex function:

$$
\varepsilon(\omega)=\varepsilon_{1}(\omega)+\mathrm{i} \varepsilon_{2}(\omega),
$$

where $\varepsilon_{1}(\omega)$ and $\varepsilon_{2}(\omega)$ are respectively the real part and the imaginary part of the dielectric function. According to the electron transition law and Kramers-Kronig transformation, we can infer the expressions as follows [31, 32]:

$$
\begin{aligned}
& \varepsilon_{1}(\omega)=1+\frac{8 \pi^{2} e^{2}}{m^{2}} \\
& \times \sum_{\mathrm{V}, \mathrm{C}_{\mathrm{BZ}}} \int_{\frac{\mathrm{d}^{3} k}{2 \pi} \frac{\left|e M_{\mathrm{CV}}(K)\right|^{2}}{E_{\mathrm{C}}(K)-K_{\mathrm{V}}(K)},} \\
& \varepsilon_{2}(\omega)=\frac{4 \pi^{2}}{m^{2} \omega^{2}} \sum_{\mathrm{V}, \mathrm{C}} \int \frac{\mathrm{d}^{3} K}{2 \pi} \\
& 2\left|e M_{\mathrm{CV}}(K)\right|^{2} \delta\left(E_{\mathrm{C}}(K)-K_{\mathrm{V}}(K)-h \omega\right),
\end{aligned}
$$

where $h$ is the Planck constant, $n$ is the reflection coefficient, $k$ is the extinction coefficient, $\omega$ is the angular frequency and $K$ is the reciprocal lattice vector. The term $e M_{\mathrm{CV}}(K)$ is the momentum transition matrix element of the transition electron, whose subscripts $\mathrm{C}$ and $\mathrm{V}$ denote the conduction and valence bands of SnSe. BZ is the first Brillouin, and $M_{\mathrm{C}}(K)$ and $M_{\mathrm{V}}(K)$ represent the intrinsic energy levels of the conduction band and valence band, respectively.

The real part of a dielectric function of pure $\mathrm{SnSe}$ and dopped SnSe systems is shown in Fig. 5a. The value of $\varepsilon_{1}(\omega)$ varies with the photon energy. Due to the effect of strain, the static dielectric function becomes larger than the unstrained SnSe, while the largest peak of $\varepsilon_{1}(\omega)$ is larger than the unstrained SnSe. Obviously, $\varepsilon_{1}(\omega)$ of SnSe is negative between $5.29 \mathrm{eV}$ and $6.91 \mathrm{eV}$, which indicates that photons in this energy range cannot travel through the medium. By observing data in Fig. 5a one can state that both the compressive strain and the tensile strain have effects on the range of negative values in the real part of the dielectric function.

Calculated values of the imaginary part of the dielectric function, i.e., $\left|\varepsilon_{2}(\omega)\right|$, are shown in Fig. 5b. The largest peak of obtained $\varepsilon_{2}(\omega)$ is observed at $2.05 \mathrm{eV}$. Comparing it with the unstrained SnSe, one can deduce that the tensile strain produce a new absorption peak at $1.91 \mathrm{eV}$ and this new peak may originate from the electron transition of Se $p$ at the bottom of the conduction band. In addition, the compressive strain produces two new absorption peaks at 0.41 and $1.97 \mathrm{eV}$. These new peaks may originate from the electron transition of Se $p$ at the top of the valence band. Now, comparing the unstrained SnSe with other different strain, we 

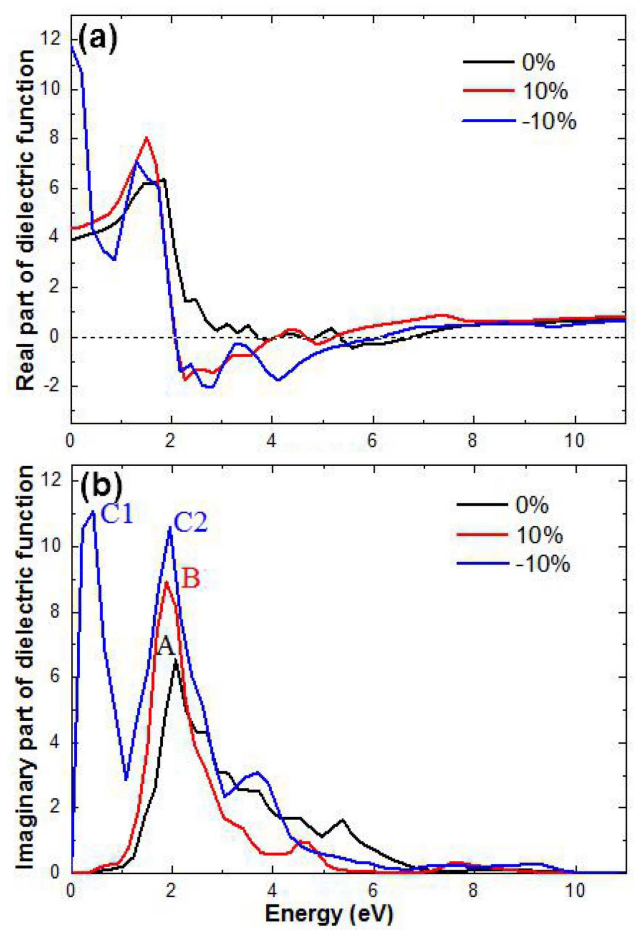

Fig. 5. The real part and imaginary part of the dielectric function of the unstrained $\delta$-SnSe, the system under the $-10 \%$ compressive strain, and the $10 \%$ tensile strain. The marks A, B, C represent the peak values of the dielectric function, whose coordinates are $\mathrm{A}(2.05,6.62), \mathrm{B}(1.91,8.94)$, $\mathrm{C} 1(0.41,11.05)$ and $\mathrm{C} 2(1.97,10.66)$, respectively.

can also note that in the low energy range dielectric absorption is more enhanced by introducing the compressive strain. In our opinion, it can be predicted that the absorption coefficient of SnSe might be enhanced in visible and infrared light range by the compressive strain.

It is noteworthy that the observed changes in electronic and optical properties might be associated with the structural distortion. For this reason, we investigate the $\mathrm{Sn}-\mathrm{Se}$ bond length, and the bond angle of both $\mathrm{Sn}-\mathrm{Se}-\mathrm{Sn}$ and $\mathrm{Se}-\mathrm{Sn}-\mathrm{Se}$, to evaluate the structural distortions (see Fig. 6). When the tensile strain is ranging from $0 \%$ to $10 \%$, the Sn1-Se2 bond length increases from $3.07 \AA$ to $3.28 \AA$, and the $\mathrm{Sn} 1-\mathrm{Se} 4$ bond length changes from $2.90 \AA$ to $2.76 \AA$ (see Fig. 6b). In the case of the Se2-Sn1-Se4 angle, its value increases from $92.87^{\circ}$ to $93.12^{\circ}$, while in the case of the Sn3-Se4-Sn1 angle, its value goes down from $87.17^{\circ}$ to $86.87^{\circ}$ (see Fig. 6c). In the meantime, under the compressive strain, ranging from $0 \%$ to $-10 \%$, one can see that the Se2-Sn1-Se4 angle is reduced from $92.87^{\circ}$ to $91.33^{\circ}$, while the Sn3-Se4-Sn1 angle increases from $87.17^{\circ}$ to $88.66^{\circ}$. Correspondingly, the Sn1-Se2 bond length increases from $3.07 \AA$ to $3.31 \AA$, and for the $\mathrm{Sn} 1-\mathrm{Se} 4$ the bond length decreases from $2.90 \AA$ to $2.76 \AA$. It is not difficult
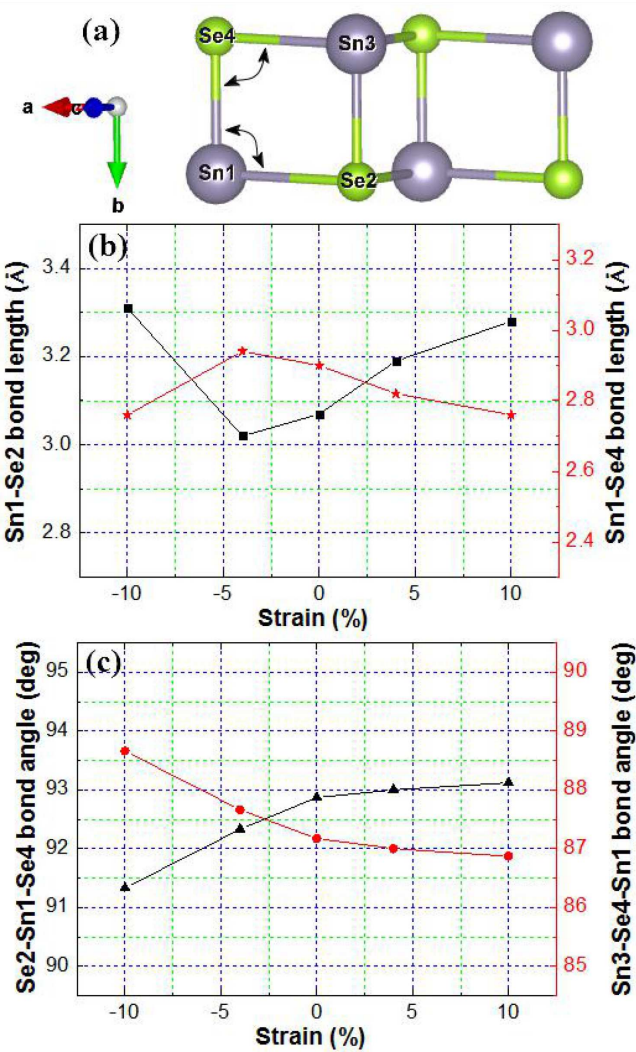

Fig. 6. The Se2-Sn1-Se4 and Sn3-Se4-Sn1 angles in $\delta$-SnSe are shown in (a), the changes of $\mathrm{Sn}-\mathrm{Se}$ bond lengths, and Se2-Sn1-Se4 and Sn3-Se4-Sn1 bond angles with different strains are given in (b) and (c).

to observe that the variations of bond lengths and bond angles are not as obvious as expected. However, it does not mean that the distortion of the geometry structure has less effect on the electronic structure and optical character of $\delta$-SnSe. Currently, we know little about this point. More work needs to be done in the future.

\section{Conclusions}

The strained $\delta$-SnSe monolayer has been systematically investigated based on first-principle calculations. The results demonstrate that the compressive strain can effectively regulate the band gap of the system. Furthermore, we calculate the dielectric function of $\delta$-SnSe under different strains, and the absorption strength in the range of visible light and infrared light has largely improved. We expect that our theoretical predictions will display functional properties in the magnetic and optical fields.

\section{Acknowledgments}

The work is supported by the Discipline Project of Shanghai Polytechnic University (Grant No. XXKZD1605), the Foundation of Shanghai Polytechnic University (Grant No. EGD18XQD29) and 
the Natural Science Foundation of Shanghai (Grant No. 19ZR1419800). Our work is also supported by the Research Center of Resource Recycling Science and Engineering, Shanghai Polytechnic University, and Gaoyuan Discipline of Shanghai-Environmental Science and Engineering (Resource Recycling Science and Engineering).

\section{References}

[1] K.S. Novoselov, A.K. Geim, S.V. Morozov, D. Jiang, Y. Zhang, S.V. Dubonos, I.V. Grigorieva, A.A. Firsov, Science 306, 666 (2004).

[2] A. K. Geim, K. S. Novoselov, Nat. Mater. 6, 183 (2007).

[3] A.H. Castro Neto, F. Guinea, N.M.R. Peres, K.S. Novoselov, A.K. Geim, Rev. Mod. Phys. 81, 109 (2009).

[4] N. Alaal, V. Loganathan, N. Medhekar, A. Shukla, J. Phys. D Appl. Phys. 49, 105306 (2016).

[5] S. Cahangirov, M. Topsakal, E. Akturk, H. Sahin, S. Ciraci, Phys. Rev. Lett. 102, 236804 (2009).

[6] D.C. Wang, L. Chen, C.M. Shi, X.L.Wang, G.L. Cui, P.H. Zhang, Y.Q. Chen, Sci. Rep. 6, 28487 (2016).

[7] R. Fei, A. Faghaninia, R. Soklaski, J.A. Yan, C. Lo, L. Yang, Nano Lett. 14, 6393 (2014).

[8] H.J. Zhang, Y.D. Ma, Z.F. Chen, Nanoscal. 7, 19152 (2015).

[9] S.L. Zhang, M.Q. Xie, F.Y. Li, Z. Yan, Y.F. Li, E.J. Kan, W. Liu, Z.F. Chen, H.B. Zeng, Angew. Chem. Int. Ed. 55, 1666 (2016).

[10] Z. Zhu, J. Guan, D. Liu, D. Tomnek, ACS Nano 9, 8284 (2015).

[11] M. Parenteau, C. Carlone, Phys. Rev. B 41, 5227 (1990).

[12] W.J. Baumgardner, J.J. Choi, Y.F. Lim, T. Hanrath, J. Am. Chem. Soc. 132, 9519 (2010).

[13] S. Yuan, Y.H. Zhu, W. Li, S. Wang, D. Xu, L. Li, Y. Zhang, X.B. Zhang, Adv. Mater. 29, 1602469 (2017).

[14] L. Li, Z. Chen, Y. Hu, X. Wang, T. Zhang, W. Chen, Q. Wang, J. Am. Chem. Soc. 135, 1213 (2013).

[15] D. Chun, R.M. Walser, R.W. Bene, T.H. Courtney, Appl. Phys. Lett. 24, 479 (1974).
[16] Z.X. Yang, G.D. Du, Q. Meng, Z.P. Guo, X. Yu, Z.X. Chen, T.L. Guo, R. Zeng, $R S C$ Adv. 1, 1834 (2011).

[17] G.J. Xiao, Y.N. Wang, J.T. Ning, Y.J. Wei, B.B. Liu, W.W. Yu, G.T. Zou, B. Zou, RSC Adv. 3, 8104 (2013).

[18] Z. Zhang, C. Shao, X. Li, L. Zhang, H. Xue, C. Wang, Y. Liu, J. Phys. Chem. C 114, 7920 (2010).

[19] X. Che, Y. Li, Y. Qu, S.R. Forrest, Nat. Energy 3, 422 (2018).

[20] G. Jeong, J. Kim, O. Gunawan, S.R. Pae, S.H. Kim, J.Y. Song, Y.S. Lee, B. Shin, J. Alloys Compd. 722, 474 (2017).

[21] M.A. Franzman, C.W. Schlenker, M.E. Thompson, R.L. Brutchey, J. Am. Chem. Soc. 132, 4060 (2010).

[22] L.C. Zhang, G. Qin, W.Z. Fang, H.J. Cui, Q.R. Zheng, Q.B. Yan, G. Su, Sci. Rep. 6, 19830 (2016).

[23] K. Zhang, K. Deng, J. Li, H. Zhang, W. Yao, J. Denlinger, Y. Wu, W. Duan, S. Zhou, Phys. Rev. Mater. 2, 054603 (2018).

[24] D.Q. Khoa, C.V. Nguyen, H.V. Phuc, V.V. Ilyasov, T.V. Vu, N.Q. Cuong, B.D. Hoi, D.V. Lu, E. Feddi, M. El-Yadri, M. Farkous, N.N. Hieu, Phys. B 545, 255 (2018).

[25] W. Guangzhao, D. Suihu, Z. Peng, X. Shuyuan, W. Cuihong, Z. Mingmin, J. Phys. D Appl. Phys. 51, 025109 (2018).

[26] D.Q. Khoa, D.T. Nguyen, C.V. Nguyen, V.T.T. Vi, H.V. Phuc, L.T.T. Phuong, B.D. Hoi, N.N. Hieu, Chem. Phys. 516 , 213 (2019).

[27] G. Kresse, J. Furthmüller, Phys. Rev. B 54, 11169 (1996).

[28] J. P. Perdew, K. Burke, M. Ernzerhof, Phys. Rev. Lett. 77, 3865 (1996).

[29] G. Kresse, D. Joubert, Phys. Rev. B 59. 1758 (1999).

[30] Z. Y. Hu, K. Li, Y. Lu, Y. Huang, X. Shao, Nanoscale 9, 16093 (2017).

[31] S. Majidi, S. M. Elahi, A. Esmailian, F. Kanjouri, Prot. Met. Phys. Chem. 53, 773 (2017).

[32] S. Majidi, N.B. Nezafat, D.P. Rai, A. Achour, H. Ghaziasadi, A. Sheykhian, S. Solaymani, Opt. Quant. Electron. 50, 292 (2018). 